\title{
Disturbance Observer based Sliding Mode Control for a Continuous Stirred Tank Reactor (CSTR)
}

\author{
MA Luning ${ }^{1}$, YNAG Xiao ${ }^{2}$ ZHAO Dongya ${ }^{1}$, SPURGEON Sarah K. ${ }^{3,1}$ \\ 1. College of Chemical Engineering, China University of Petroleum, Qingdao 266555, P. R. China \\ E-mail: dyzhao@upc.edu.cn; dongyazhao@139.com \\ 2. School of Automation, Beijing Institute of Technology, Beijing 100081, P. R. China \\ 3. Department of Electronic \& Electrical Engineering, University College London, Torrington Place, London WC1E 7JE, United Kingdom \\ E-mail: s.spurgeon@ucl.ac.uk
}

\begin{abstract}
A continuous stirred tank reactor (CSTR) is typical of equipment found in the process control industry. The dynamics represent a wide class of second order nonlinear systems and thus as well as having specific industrial application, control of the CSTR is frequently used as a benchmark problem for application and testing of new control algorithms. Due to the high complexity of the CSTR system, the robust control design problem is challenging. This paper first establishes a mathematical model of the system. A disturbance observer is then designed to estimate the disturbance and a corresponding asymptotically stable sliding mode control is developed. Stability analysis is presented in terms of the Lyapunov method. Finally, based on experimental data, the proposed method is validated using simulation experiments.
\end{abstract}

Key Words: CSTR, disturbance observer, sliding mode control

\section{Introduction}

The chemical industry is an important pillar of the national economy, and the continuous stirred tank reactor (CSTR), which has complex, time-varying and nonlinear characteristics is a key element of equipment required to complete chemical reactions. Research work investigating the advanced control of a CSTR thus has significant impact on both control theory and application.

Numerous control methods have been applied for control of the CSTR, such as PID control, fuzzy control and adaptive control. However these control methods have many shortcomings, which may include poor performance, complex design or the requirement for the control to be implemented with a human in the loop. In contrast, sliding mode control is known to possess inherent robustness properties. In the sliding mode, the closed-loop response becomes totally insensitive to a particular class of system uncertainty. This class of uncertainty is called matched uncertainty and is categorised by uncertainty that is implicit in the input channels. There are many research directions in the area of sliding mode control [1], which include quasi-sliding mode control [2], sliding mode control based on a reaching law [3], sliding mode control for discrete systems[4], terminal sliding mode control [5-7] and integral sliding mode control [8]. The paradigm is suitable for linear and nonlinear systems, certain and uncertain systems, and more and more practical implementations are being reported in diverse sectors, such as robot control [9], aircraft control [10] and industrial process control [11]. A key feature of the sliding mode control approach is the ability to specify desired plant dynamics by choice of the switching function. A control law must then be defined to guarantee the existence of a sliding motion i.e. to ensure the state or output of the plant is attracted to the desired dynamics. For example, in [12], a terminal sliding mode control is designed for uncertain systems, and

This work is partially supported by the National Nature Science Foundation of China under Grant 61473312 and the Changjiang Scholar Project. good control performance is achieved. Defining the sliding mode dynamics by selecting an appropriate sliding surface is termed as solving the existence problem. A control to ensure the desired sliding mode dynamics are attained and maintained is sought by means of solving the reachability problem. For time-varying, strongly non-linear reactor dynamics, the sliding mode control paradigm is seen to be a good candidate for controller design given the inherent insensitivity to parameter changes and uncertainty, which means that detailed system identification is not required to obtain a highly accurate model for the design purpose and robustness is exhibited at implementation. The method can also ensure a fast response [13].

For systems exhibiting external disturbances and parameter uncertainty, a variety of control methods [14-16] have been developed to improve the robustness and performance of systems. Among these methods, a disturbance observer (DO) is widely used in the design of servo control systems [17-19] due to its simple structure and ability to improve tracking accuracy. Linear and nonlinear representations of disturbance observers have been developed. The non-linear disturbance observer (NDO) has been found to exhibit better performance and has been widely used. NDO methods include a fuzzy disturbance observer [20], sliding mode disturbance observer [21] and neural network disturbance observer [22]. In [23], a disturbance observer is designed for a manipulator. Good disturbance approximation is achieved and the disturbance effect is effectively reduced.

Inspired by the literature [24], this paper will establish a CSTR dynamic model. Then, a disturbance observer will be designed to estimate the external disturbance present in the controlled CSTR system. Based on sliding mode control theory, a new control algorithm is designed to realize disturbance observation and asymptotic stability of the CSTR system. Note that it is possible to design a sliding mode controller without using the disturbance estimate. However, a conservative control design will result; the switched control element would be required to have much higher gain in 


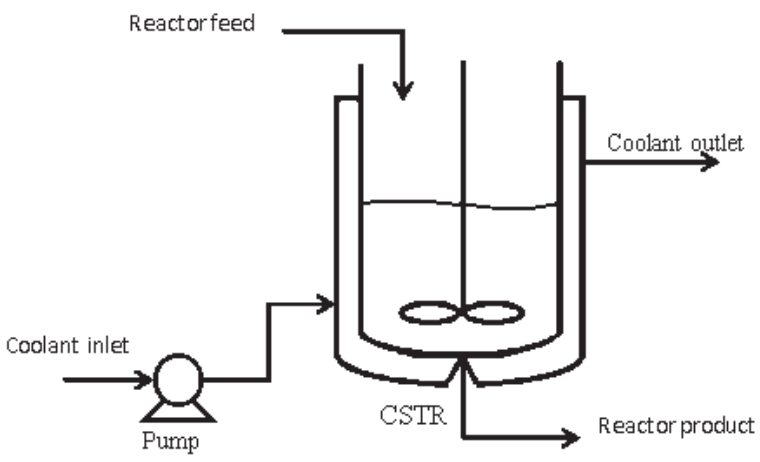

Fig. 1: The model of the CSTR.

this case as the magnitude of the switch in the sliding mode control would have to be selected to accommodate the worst case disturbance signal characteristics.

\section{CSTR Model Analysis}

In this paper, the case of a CSTR with cooling fluid in the jacket is studied. It is assumed that the volume of the continuous stirred tank reactor is $V$ and that unreacted material is added to the CSTR with temperature $T_{f}$. The corresponding concentration is $C_{A f}$, the density is $\rho$ and the flow rate is $q$. In the tank, a first-order exothermic irreversible reaction $A \rightarrow B+\Delta H$ is assumed. After the reaction, the temperature of the outlet material is $T$, the concentration is $C_{A}$ and the flow rate is $q$. The temperature of the coolant in the jacket is $T_{c}$ and the flow rate is $q_{c}$. The flow rate of the coolant in the jacket $q_{c}$ is selected as the manipulated variable or control, and the outlet concentration $C_{A}$ as well as the temperature $T$ in the reactor are selected as the two state variables. The required reactant concentration $C_{A}$ and the temperature $T$ in the reactor are obtained by varying the amount of coolant flow $q$.

Assume that the model can achieve the following requirements [25]:

(1) The total volume of the material in the reactor is unchanged before the start and after the end of the reaction;

(2) The material in the reactor is thoroughly mixed;

(3) The reaction material has constant density and properties.

According to the conservation of material and energy conservation, the following CSTR dynamic model can be established:

$$
\begin{aligned}
\dot{C}_{A}= & \frac{q}{V}\left(C_{A f}-C_{A}\right)-k_{0} C_{A} e^{-E / R T} \\
\dot{T}= & \frac{q}{V}\left(T_{f}-T\right)+\frac{(-\Delta H)}{\rho C_{p}} k_{0} C_{A} e^{-E / R T}+ \\
& \frac{U A}{\rho V C_{p}}\left(T_{c}-T\right)
\end{aligned}
$$

where $k_{0}$ is the exponential factor, $E$ is the activity energy, $R$ is the gas constant, $T$ is the temperature, $M$ is the mass, $C_{p}$ is the specific heat, $\rho$ is the material density, $\Delta H$ is the reflect heat enthalpy, $U$ is the heat transfer coefficient and $A$ is the heat transfer area.

To transfer equation (1) into a general form for controller design, the following change of variables is used:

$$
\begin{aligned}
& a=q / V, b=U A / r V C_{p}, \quad \gamma=E / R T_{f 0}, \\
& b=\Delta H C_{A f} / C_{p} T_{f 0} \rho, \quad D_{a}=k_{0} \exp (-\gamma), \\
& x_{1}=C_{A f}-C_{A} / C_{A f}, \quad x_{2}=\left(T-T_{f 0}\right) \gamma / T_{f 0}, \\
& u=\gamma\left(T_{c}-T_{f 0}\right) / T_{f 0}, d=\left(T_{f}-T_{f 0}\right) / T_{f 0}
\end{aligned}
$$

Using the above dimensionless formulae, the nonlinear dynamic model of the CSTR system can be rewritten as

$$
\begin{aligned}
\dot{x}_{1} & =-a x_{1}+D_{a}\left(1-x_{1}\right) e^{\gamma x_{2} / \gamma+x_{2}} \\
\dot{x}_{2} & =-a x_{2}-b D_{a}\left(1-x_{1}\right) e^{\gamma x_{2} / \gamma+x_{2}}+\beta\left(u-x_{2}\right)+d \\
y & =x_{2}
\end{aligned}
$$

The temperature $x_{2}$ of the CSTR system is often selected as an important indicator of the system output response in practice.

\section{Nonlinear Disturbance Observer}

Uncertainties and external disturbances affect the CSTR system. In order to reduce these effects and improve the control performance, a nonlinear disturbance observer is designed inspired by the work in [26] to estimate the uncertainty and the disturbance. This nonlinear disturbance observer has the advantage of having a simple structure, fast response and very accurate tracking performance.

To facilitate observer design, the CSTR representation in (2) will be rewritten in the following vector form:

$$
\begin{aligned}
& \dot{x}_{1}=f_{1}\left(x_{1}, x_{2}\right) \\
& \dot{x}_{2}=f_{2}\left(x_{1}, x_{2}\right)+\beta u+d
\end{aligned}
$$

where

$$
\begin{aligned}
& f_{1}\left(x_{1}, x_{2}\right)=-a x_{1}+D_{a}\left(1-x_{1}\right) e^{\gamma x_{2} / \gamma+x_{2}} \\
& f_{2}\left(x_{1}, x_{2}\right)=-a x_{2}-b D_{a}\left(1-x_{1}\right) e^{\gamma x_{2} / \gamma+x_{2}}-\beta x_{2}
\end{aligned}
$$

The state equation of the CSTR system can be conveniently rewritten as:

$$
\dot{x}=F(x)_{2 \times 1}+G(x)_{2 \times 2} u_{2 \times 1}+D_{2 \times 1}
$$

where from (3), $x=\left[x_{1}, x_{2}\right]^{T}, \quad F(x)=$ $\left.\left[f_{1}\left(x_{1}, x_{2}\right), f_{2}\left(x_{1}, x_{2}\right)\right)\right]^{T}, D=[0, d]^{T}, u=\left[0, u_{c}\right]^{T}$, $G(x)=\left[\begin{array}{ll}0 & 0 \\ 0 & \beta\end{array}\right]$.

Define the corresponding nonlinear disturbance observer as:

$$
\begin{aligned}
\hat{D} & =z+P \\
\dot{z} & =-L z-L(P+F(x)+G(x) u)
\end{aligned}
$$

where $\hat{D}$ is the output of the disturbance observer, $z$ is the disturbance observer internal variable, $P$ is a design function, $L=\operatorname{diag}[c, c]$ is a gain matrix and $c>0$ is a real number. Define the function $P$ as follows:

$$
\dot{P}=L \dot{x}
$$

From (4) and (5) the disturbance observer error is defined as:

$$
\tilde{D}=D-\hat{D}
$$

Assume the disturbance is slowly varying so that $\dot{D}=0$. Then the error dynamics are given by:

$$
\begin{aligned}
\dot{\tilde{D}} & =-\dot{\hat{D}}=-\dot{z}-\dot{P} \\
& =L z+L(P+F(x)+G(x) u))-L \dot{x} \\
& =L \hat{D}-L D=-L \tilde{D}
\end{aligned}
$$


Therefore, for each controlled system state, the observer error equation is obtained as:

$$
\dot{\tilde{D}}_{i}+L_{i} D_{i}=0
$$

Solving the ordinary differential equation (9):

$$
\tilde{D}_{i}(t)=\tilde{D}_{i}\left(t_{0}\right) \mathrm{e}^{-L_{i} t}
$$

where $\tilde{D}_{i}\left(t_{0}\right)$ is the initial value of $\tilde{D}_{i}(t)$. It is clear that when time $t$ approaches infinity, $\tilde{D}_{i}(t)$ is equal to zero and the disturbance observer error $\tilde{D}_{i}(t)$ is asymptotically stable.

Consider now the case when there is a time varying external disturbance, but the rate of change is limited, so that the derivative of the disturbance is bounded:

$$
\left|\dot{D}_{i}\right| \leq \alpha
$$

Considering equation (8), the derivative of $\tilde{D}$ is:

$$
\dot{\tilde{D}}_{i}=\dot{D}_{i}-\dot{\hat{D}}_{i}=\dot{D}_{i}-L_{i} \dot{\tilde{D}}_{i}
$$

Solving the differential equation (12), it is obtained that:

$$
\tilde{D}_{i}(t)=D(0) e^{-L_{i} t}+e^{-L_{i}} \int_{0}^{t} \dot{D}_{i} e^{L_{i} t} d t
$$

When $\dot{D}_{i} \leq \alpha$, it follows that:

$$
\begin{aligned}
\tilde{D}_{i}(t) & \leq D_{i}(0) e^{-L_{i} t}+e^{-L_{i}} \alpha \int_{0}^{t} e^{L_{i} t} d t \\
& =\left(D_{i}(0)-\frac{\alpha}{L_{i}}\right) e^{-c t}+\frac{\alpha}{L_{i}}
\end{aligned}
$$

and

$$
\tilde{D}_{i}(\infty) \leq \frac{\alpha}{L_{i}}
$$

When $\dot{D} \geq \alpha$, the following equation can be obtained:

$$
\tilde{D}_{i}(t) \geq\left(D_{i}(0)+\frac{\alpha}{L_{i}}\right) e^{-L_{i} t}-\frac{\alpha}{L_{i}}
$$

and it follows that

$$
\tilde{D}_{i}(\infty) \geq-\frac{\alpha}{L_{i}}
$$

Therefore, the estimation error is bounded. Define the bound as:

$$
\left|\tilde{D}_{i}\right| \leq \varsigma
$$

where $\varsigma=\frac{\alpha}{L_{i}}$.

Lemma 1 By proper design of the matrix $L$, the nonlinear disturbance observer error will converge exponentially into a closed sphere of finite radius, and the radius of the sphere can be made sufficiently small.

Proof: Choose the following Lyapunov function candidate:

$$
V_{0}=0.5 \tilde{D}_{i}^{2}
$$

Differentiating $V_{0}$ with respect to time yields

$$
\begin{aligned}
\dot{V}_{0} & =\tilde{D}_{i}\left(\dot{D}_{i}-\dot{\hat{D}}_{i}\right)=\tilde{D}_{i}\left(\dot{D}_{i}-L_{i} \tilde{D}_{i}\right) \\
& \leq\left(\dot{D}_{i}-L_{i} \tilde{D}_{i}\right)\left|\tilde{D}_{i}\right| \\
& \leq-\left(L_{i} \tilde{D}_{i}-\alpha\right)\left|\tilde{D}_{i}\right|
\end{aligned}
$$

Therefore, the disturbance observer error $\tilde{D}_{i} \leq \alpha / L_{i}$ is obtained, and the range of the observation error is sufficiently small if the gain matrix $L$ is reasonably designed.

\section{Sliding mode control design}

The integral sliding mode control (ISMC) approach exhibits strong robustness, as effectively the reaching phase present in classical sliding mode controller design is eliminated [27-29] and good results have been achieved in applications including servo motor control and robot control. This paper adopts the integral form of sliding surface as in the literature for the CSTR [30].

The dynamic equation of the CSTR system is taken from (2) and the system tracking error $e$ is defined as:

$$
e=x_{2}-x_{d}
$$

where $x_{d}$ is the desired system output.

Define the switching function as:

$$
s=e+\lambda \int_{0}^{t} e(\tau) d \tau
$$

By differentiating (22) with respect to time yields:

$$
\begin{aligned}
\dot{s}= & \dot{e}+\lambda e \\
= & -a x_{2}-b D_{a}\left(1-x_{1}\right) e^{\gamma x_{2} / \gamma+x_{2}}+\beta u- \\
& \beta x_{2}+d+\lambda e
\end{aligned}
$$

Assume a sliding motion has been reached and $\dot{s}=0$. The corresponding so called equivalent control $u_{e q}$ can be obtained as:

$$
\begin{aligned}
u_{e q}= & -\frac{1}{\beta}\left[-a x_{2}-b D_{a}\left(1-x_{1}\right) e^{\gamma x_{2} / \gamma+x_{2}}-\right. \\
& \left.\beta x_{2}+d+\lambda e\right]
\end{aligned}
$$

where $\lambda>0$ is a real number. The equivalent control represents the effective control action which is necessary to maintain the ideal sliding motion. It is not the control action directly applied to the plant but can be thought of as representing, on average, the effect of the applied discontinuous control required to maintain a sliding mode. In the case of the CSTR, the equivalent control as stated in (24) cannot be applied as it is a function of the disturbance which is not measured. In this paper the disturbance estimate from the DO will be used in the equivalent control. An augmenting switching control is defined as:

$$
u_{s}=-\eta \operatorname{sgn}(s)
$$

where $\operatorname{sgn}(s)$ is the sign function and $\eta>0$ is a real number. The corresponding control law is chosen as:

$$
u=u_{e q}+u_{s}
$$

where it is important to note that $u_{e q}$ is computed using the disturbance estimate.

Theorem 1 If the controller is consistent with (26) and the disturbance estimate is in the form of (5), then the closedloop control system is asymptotically stable.

Proof: Choose a Lyapunov function candidate as:

$$
V=0.5 s^{2}
$$


Differentiating $V$ with respect to time yields:

$$
\begin{aligned}
\dot{V} & =s \dot{s} \\
& =s(-\hat{D}+D-\eta \operatorname{sign}(s)) \\
& \leq-\eta|s|+\varsigma|s| \\
& \leq-(\eta-\varsigma)|s|<0
\end{aligned}
$$

Select the parameter $\eta>\varsigma$ so that the sliding condition is satisfied, then the system state will reach the sliding mode in finite time. After the system state reaches the sliding mode, it will approach zero as time approaches infinity.

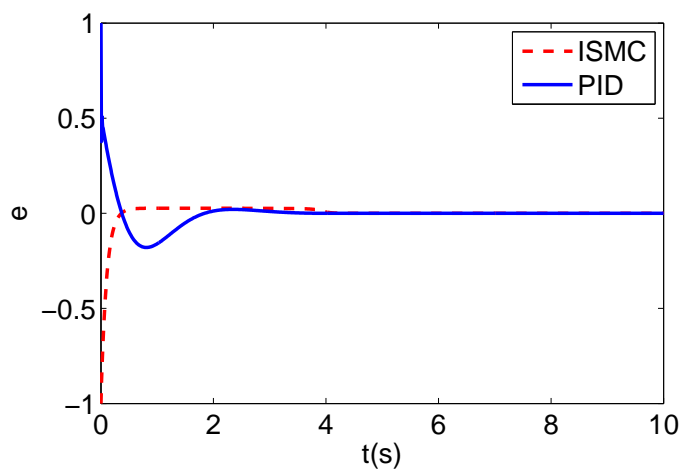

Fig. 2: Comparison of the error curves between a PID control and the proposed sliding mode control system in the absence of any external disturbance

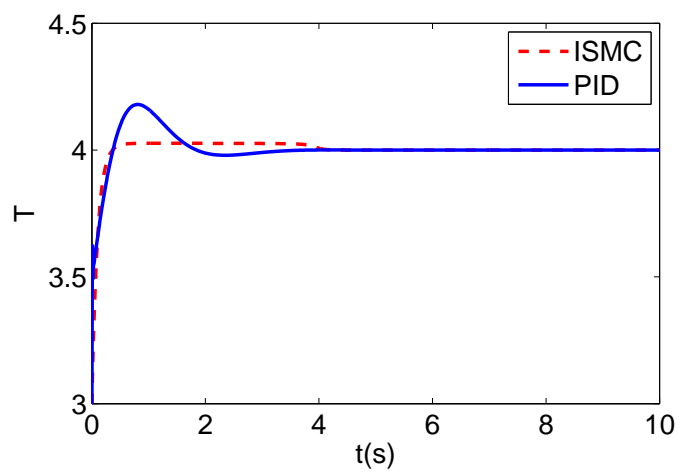

Fig. 3: Comparison of the temperature curve between a PID control and the proposed sliding mode control system in the absence of any external disturbance

\section{Simulation analysis}

The proposed approach is now validated by using Matlab/Simulink experiments and the corresponding simulation results are analyzed. The dynamic equation of the CSTR is described by (2), where the parameters $a=1.0, \beta=0.3$, $\gamma=20, b=8, D_{a}=0.072$. In the simulation, the initial value of the concentration is $x_{1}(0)=0.5$, the initial value of the temperature is $x_{2}(0)=3$, and the output of the system is expected to be $y_{r}=4$.

In the simulation analysis, the performance of the designed control is compared with that of a classical PID control. Initially no external disturbance is applied. The results are shown in Figure 2 and Figure 3. The performance in the presence of disturbances is now considered and the designed disturbance observer is first tested. Setting the external disturbance to $d=\sin (5 t)$, the output of the disturbance observer is shown in Figure 4. Figure 5 and Figure 6 show the temperature tracking and tracking error when the sliding mode control is used without the DO and with the DO, respectively.

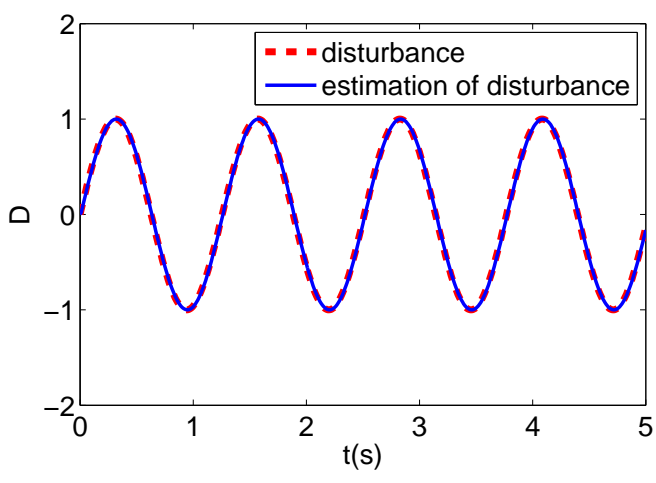

Fig. 4: The output of disturbance observer

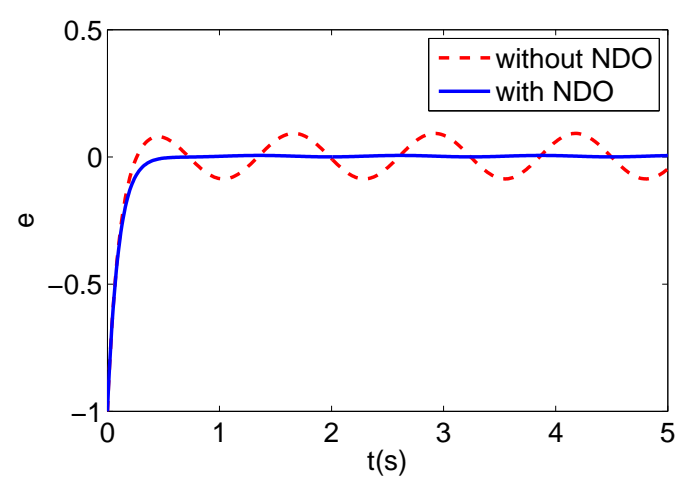

Fig. 5: Comparison of the error curves for the sliding mode control with disturbance observer system and a sliding mode control where the disturbance observer is not used and the magnitude of the discontinuous element is used to counteract the effect of the disturbance, Note that $\eta$ is fixed

It can be seen from Figure 3 that compared to traditional PID control, the sliding mode controller reduces the temperature overshoot significantly and the speed of response is greatly improved. It can be seen from Figure 4 that the disturbance observer rapidly tracks the external disturbance. From Figure 5 and Figure 6 it is seen that the closed-loop CSTR system based on the disturbance observer is more robust. It should be noted that it is possible to increase the value of $\eta$ in the discontinuous control component to provide better performance when the DO is not used to estimate the disturbance for use by the controller. However, the corresponding control would be more conservative and less energy efficient. Figures 7 and 8 compare the error and 


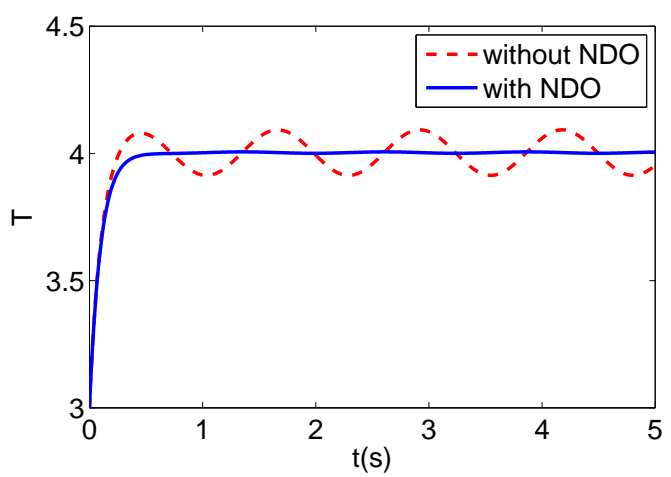

Fig. 6: Comparison of temperature curves between disturbance observer system and non-disturbance observer system for fixed $\eta$

temperature trajectories when the sliding mode controller is implemented with a disturbance observer and discontinuous control gain of $\eta=0.1$ and the sliding mode controller is implemented without the disturbance observer and the discontinuous control gain is increased to $\eta=5$. It is clear that in terms of both the transient and steady state performance the sliding mode controller and disturbance observer system is superior. Figure 9 shows the corresponding control signal and it is seen that use of the disturbance observer reduces the required control effort and, as well as providing superior tracking performance, also produces a more energy efficient control strategy.

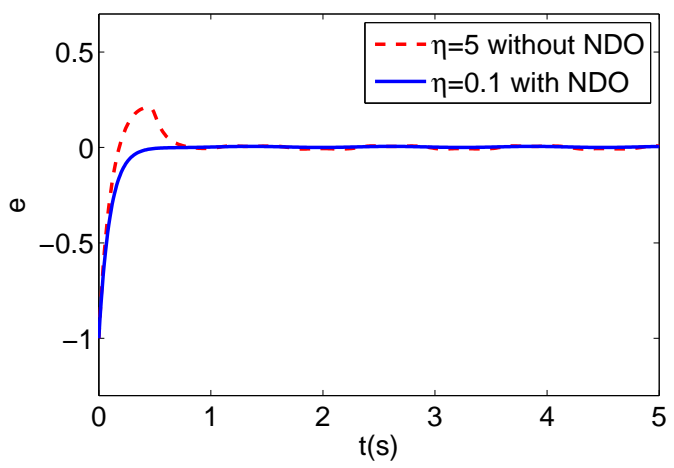

Fig. 7: Comparison of the error curves for the sliding mode control with disturbance observer system and a sliding mode control where the disturbance observer is not used and the magnitude of the discontinuous element is increased to counteract the effect of the disturbance

\section{Conclusion}

A dynamic model of a CSTR has been described and an asymptotically stable closed-loop control established by combining a sliding mode controller and a nonlinear disturbance observer. It has been shown that by selecting appropriate design parameters, the nonlinear disturbance observer can exhibit a rapid rate of convergence and reduce tracking error. This disturbance observer is combined with an integral sliding mode controller and the stability of the resulting closed-loop system is proved by using the Lyapunov method.
Simulation results show that the design approach exhibits good disturbance rejection, exhibiting improved robustness and reducing the tracking error when compared with a classical control design. Future work will consider the practical implementation of the proposed design on an experimental test facility.

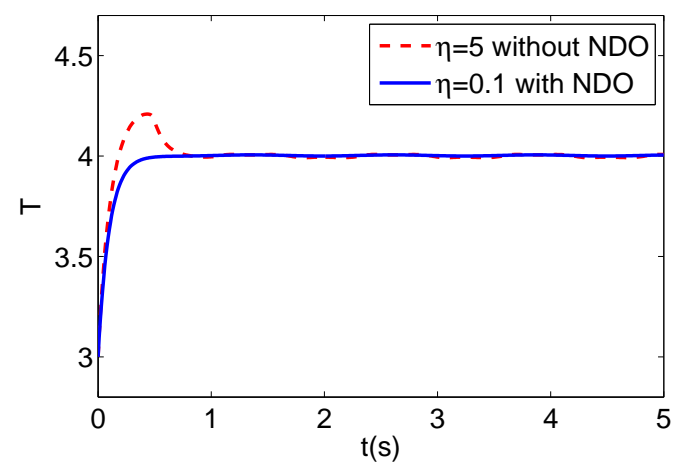

Fig. 8: Comparison of the temperature curves for the sliding mode control with disturbance observer system and a sliding mode control where the disturbance observer is not used and the magnitude of the discontinuous element is increased to counteract the effect of the disturbance

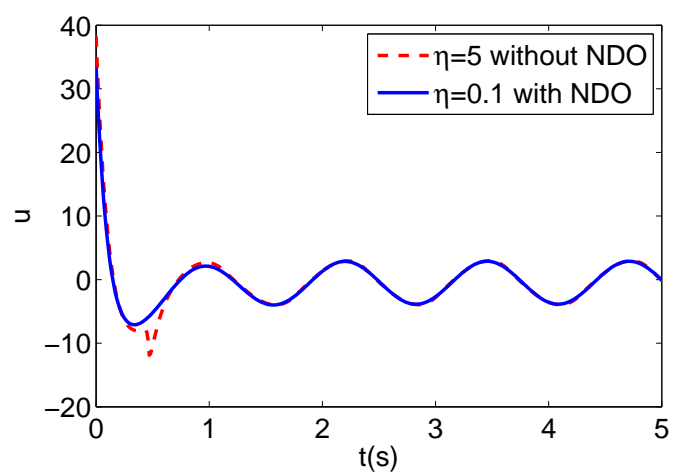

Fig. 9: Comparison of the control signal for the sliding mode control with disturbance observer system and a sliding mode control where the disturbance observer is not used and the magnitude of the discontinuous element is increased to counteract the effect of the disturbance

\section{References}

[1] J.-K. Liu and F.-C. Sun, "Research and development on theory and algorithms of sliding mode control." Kongzhi Lilun yu Yingyong/ Control Theory \& Applications, vol. 23, no. 3, pp. 407-418, 2007.

[2] J.-J. Slotine and S. S. Sastry, "Tracking control of non-linear systems using sliding surfaces, with application to robot manipulators" International Journal of Control, vol. 38, no. 2, pp. 465-492, 1983.

[3] W. Gao, "Theory foundation of variable structure control," Science and Technology Press of China, 1990.

[4] W. Gao, Y. Wang, and A. Homaifa, "Discrete-time variable structure control systems," IEEE Transactions on Industrial Electronics, vol. 42, no. 2, pp. 117-122, 1995. 
[5] M. Zhihong, A. P. Paplinski, and H. R. Wu, "A robust mimo terminal sliding mode control scheme for rigid robotic manipulators," IEEE Transactions on Automatic Control, vol. 39, no. 12, pp. 2464-2469, 1994.

[6] X. Yu and Z. Man, "Model reference adaptive control systems with terminal sliding modes," International Journal of Control, vol. 64, no. 6, pp. 1165-1176, 1996.

[7] M. Zhihong and X. H. Yu, "Terminal sliding mode control of MIMO linear systems," in Decision and Control, 1996., Proceedings of the 35th IEEE Conference on, vol. 4. IEEE, 1996, pp. 4619-4624.

[8] J.-D. Wang, T.-L. Lee, and Y.-T. Juang, "New methods to design an integral variable structure controller," IEEE Transactions on Automatic Control, vol. 41, no. 1, pp. 140-143, 1996.

[9] H. M. Becerra, G. López-Nicolás, and C. Sagüés, "A slidingmode-control law for mobile robots based on epipolar visual servoing from three views," IEEE Transactions on Robotics, vol. 27, no. 1, pp. 175-183, 2011.

[10] J. Zhao, B. Jiang, P. Shi, and H. Liu, "Adaptive dynamic sliding mode control for near space vehicles under actuator faults," Circuits, Systems, and Signal Processing, vol. 32, no. 5, pp. 2281-2296, 2013.

[11] X.-H. Xie, Y.-F. Dai, and S.-Y. Li, "Fuzzy sliding mode controller for servo tracking control in precision machine tools." Kongzhi Lilun yu Yingyong/Control Theory \& Applications(China), vol. 20, no. 6, pp. 913-918, 2003.

[12] Y. Wu, X. Yu, and Z. Man, "Terminal sliding mode control design for uncertain dynamic systems," Systems \& Control Letters, vol. 34, no. 5, pp. 281-287, 1998.

[13] G. Weibing, "Theory and design method of variable structure control," Science and Technology Press, Beijing, 1996.

[14] B.-K. Choi, C.-H. Choi, and H. Lim, "Model-based disturbance attenuation for cnc machining centers in cutting process," IEEE/ASME Transactions on Mechatronics, vol. 4, no. 2, pp. $157-168,1999$.

[15] B. Yao, M. Al-Majed, and M. Tomizuka, "High-performance robust motion control of machine tools: an adaptive robust control approach and comparative experiments," IEEE/ASME Transactions on Mechatronics, vol. 2, no. 2, pp. 63-76, 1997.

[16] T. Zhang, J. Zhou, and J. Guo, "Design of predictive controller for hypersonic vehicles based on disturbance observer," Acta Aeronautica et Astronautica Sinica, vol. 1, p. 022, 2014.

[17] Z. Shaode and C. Zhucheng, "Design method for servosystem based on disturbance observer," Journal of UEST of China, vol. 34, no. 1, pp. 85-88, 2005.

[18] H.-Y. Wang, Q.-L. Wang, Z. Zuo, J.-H. Qiao, and Y.-H. Xia,
"Sliding mode variable structure control for tank servo system based on disturbance observer," Journal of System Simulation, vol. 14, p. 067, 2009.

[19] Y. F. Huang, Y. J. Wu, and B. T. Liu, "Research on internal model control method based on disturbance observer for high performance turntable servo system," Journal of System Simulation, vol. 23, no. 8, pp. 1664-1667, 2011.

[20] M. Pu, Q. Wu, C. Jiang, and L. Cheng, "Adaptive second-order dynamic sliding-mode control based on fuzzy disturbance-observer," Kongzhi Lilun Yu Yinyong/control Theory and Applications, vol. 28, no. 6, 2011.

[21] Y. Ren, "Application of low speed opto-electronic tracking systems based on sliding mode distutbance observer," Journal of Beijing University of Aeronautics and Astronautics, vol. 39, no. 6, pp. 835-840, 2013.

[22] M. Chen, Q. Zou, C. Jiang, and Q. Wu, "Dynamical inversion flight control based on neural network disturbance observer," Kongzhi Yu Juece/control and Decision, vol. 23, no. 3, pp. 283287, 2008.

[23] W. H. Chen, D. J. Ballance, P. J. Gawthrop, and J. O'Reilly, "A nonlinear disturbance observer for robotic manipulators," IEEE Transactions on Industrial Electronics, vol. 47, no. 4, pp. 932-938, 2000.

[24] J. Yu, M. Chen, and C. S. Jiang, "Adaptive sliding mode control for nonlinear uncertain systems based on disturbance observer," Control Theory and Applications, 2014.

[25] J. Albert, "Fast model predictive control," Ph.D. dissertation, University of Oxford, 2013.

[26] J. Liu and X. Wang, Advanced Sliding Mode Control for Mechanical Systems. Springer Berlin Heidelberg, 2012.

[27] T. L. Chern and Y. C. Wu, "Design of integral variable structure controller and application to electrohydraulic velocity servosystems," IEE Proceedings D, vol. 138, no. 5, pp. 439-444, 1991.

[28] T. L. Chern and Y. C. Wu, "An optimal variable structure control with integral compensation for electrohydraulic position servo control systems," IEEE Transactions on Industrial Electronics, vol. 39, no. 5, pp. 460-463, 1992.

[29] T. L. Chern and J. S. Wong, "Dsp based integral variable structure control for dc motor servo drivers," Control Theory and Applications, IEE Proceedings -, vol. 142, no. 5, pp. 444450, 1995.

[30] D. Zhao, Q. Zhu, and J. Dubbeldam, "Terminal sliding mode control for continuous stirred tank reactor," Chemical Engineering Research and Design, vol. 94, pp. 266-274, 2015. 